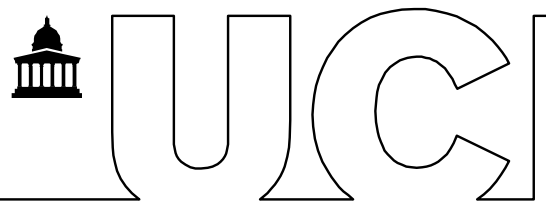

Lawson, B; Franklinos, L; Efstratiou, A; Macgregor, SK; Hopkins, T; Cunningham, AA; Streptococcus pyogenes infection in a free-living European hedgehog (Erinaceus europaeus). Ecohealth 10.1007/s10393-015-1051-2. (In press). Downloaded from UCL Discovery:

http://discovery.ucl.ac.uk/1460770

\title{
ARTICLE
}

\section{Streptococcus pyogenes infection in a free-living European hedgehog (Erinaceus europaeus)}

\author{
Lydia H. V. Franklinos ${ }^{1}$, Androulla Efstratiou', Shaheed K. Macgregor ${ }^{3}$, Shinto K. John ${ }^{1}$, \\ Timothy Hopkins ${ }^{1}$, Andrew A. Cunningham ${ }^{1}$, Becki Lawson ${ }^{1}$ \\ ${ }^{1}$ Institute of Zoology, Zoological Society of London (affiliated with University College \\ London) \\ ${ }^{2}$ Public Health England, Colindale, London \\ ${ }^{3}$ Living Collections, Zoological Society of London (affiliated with University College London)
}

\begin{abstract}
Streptococcus pyogenes, a common pathogen of humans, was isolated from the carcass of a free-living European hedgehog (Erinaceus europaeus) found in northern England in June 2014. The animal had abscessation of the deep right cervical lymph node, mesenteric lymph nodes and liver. The $S$. pyogenes strain isolated from the lesions, peritoneal and pleural cavities was characterised as emm 28, which can be associated with invasive disease in humans. This is the first known report of $S$. pyogenes in a hedgehog and in any free-living wild animal that has been confirmed by gene sequencing. As close associations between wild hedgehogs and people in England are common, we hypothesise that this case might have resulted from anthroponotic infection.
\end{abstract}

Streptococcus pyogenes (Lancefield group A streptococcus; [GAS]) is a Gram-positive facultative anaerobic coccus which is the main cause of bacterial pharyngitis in humans as well as causing severe invasive disease, such as streptococcal toxic shock syndrome (Cunningham 2000). The rate of GAS infections reported in 2006 from England, Wales and Northern Ireland was 3.3 per 100,000 population, with the highest levels in the young and the elderly (Lamagni et al., 2008).

The European hedgehog (Erinaceus europaeus) has a range that extends across Europe and is widely distributed in Great Britain despite recent dramatic population declines (Harris et al., 1995; Wembridge, 2011). Relatively little is known about the infectious diseases of hedgehogs, but there have been reports of potentially zoonotic infections in this species including Salmonella spp., Borrelia spp., Cryptosporidium sp. and mecC-positive Staphylococcus aureus (Meredith and Milne, 2009; Riley and Chomel, 2005; Skuballa, et al., 2007; Widen et al., 1996).

A wild hedgehog was found dead in June 2014 within a suburban garden in Northumberland, England. Wild hedgehogs had been regularly observed at the site but were not provided with supplementary food, although this could have occurred in neighbouring properties. The garden was frequented by five apparently healthy domestic cats. No other domestic or livestock species were reported in the immediate vicinity. No previous sign of ill-health was seen in any wild hedgehog prior to finding the carcass of the 
submitted animal. A post-mortem examination was conducted consisting of systematic external and internal inspection of all body systems, combined with microbiological and histopathological examinations, as described below.

The hedgehog was an adult male and was in poor body condition with severe, diffuse periodontal disease and an oronasal fistula connecting the empty socket of the upper right first incisor with the right nasal cavity.

Several tissues, including the liver, the right deep cervical lymph node and multiple mesenteric lymph nodes, contained multiple abscesses containing yellow turbid fluid. Aseptically collected tissue samples were plated onto each of the following media: (a) Columbia blood agar supplemented with $5 \%$ horse blood (CBA) (Oxoid Ltd., Basingstoke, UK) incubated under aerobic, anaerobic conditions and in air supplemented with $\mathrm{CO}_{2}$; (b) Columbia agar with chocolate horse blood (Oxoid Ltd.) incubated in air supplemented with $\mathrm{CO}_{2}$; (c) Xylose-Lysine Deoxycholate (XLD) agar (Oxoid Ltd.) incubated under aerobic conditions. All culture media were incubated at $37^{\circ} \mathrm{C}$ and reviewed at day one, two and five. Samples from the right deep cervical lymph node, hepatic abscess, peritoneal cavity and pleural cavity yielded a pure, confluent growth of small $(<1 \mathrm{~mm}$ diameter), translucent, entire, butyrous, beta-hemolytic colonies on the CBA plate after 48 hours of anaerobic incubation. The isolate, a Gram-positive coccus, was both oxidase-and catalyse-negative and was identified as a beta-hemolytic Streptococcus sp. Lancefield group A using a Prolex TM Lancefield latex agglutination test (Pro-Lab Diagnostics Ltd., Wirral, UK). The isolate was further identified as $S$. pyogenes using API 20 Strep identification strip (bioMérieux UK Ltd.) (API 20 Streptococcus profile 0160514). Using the CDC emm sequencing protocol (http://www.cdc.gov/streplab/protocol-emm-type.html), this was characterized as an emm 28 strain. No other significant microbial isolates were obtained.

Samples from brain, heart, kidney, liver, lung, spleen, trachea, small and large intestine, the right cervical lymph node were fixed in $10 \%$ neutral buffered formalin, embedded in paraffin, sectioned and stained with haematoxylin and eosin, Gram's, Ziehl-Neelsen and Periodic acid-Schiff stains using routine methods. Histopathological examination of the liver showed diffuse congestion, hemorrhagic foci, loss of architecture and multiple focal-to-coalescing areas of acute hepatocellular necrosis with co-localization of Gram-positive cocci. Microscopic examination of the right deep cervical lymph node showed this to contain a mixed infiltrate of degenerative neutrophils and lymphocytes and multiple focal-to-coalescing areas of acute necrosis. No abnormalities were detected on examination of the remaining tissues. No additional organisms were detected on histological examination.

We identified disseminated $S$. pyogenes infection as the cause of death of a wild hedgehog in Britain. The animal had periodontal disease, which is commonly seen in wild hedgehogs (Robinson and Routh, 1999) and a tooth root abscess. These lesions might have been the site of entry for the bacterium, from where it spread to other tissues. To the best of our knowledge, this is the first report of $S$. pyogenes infection in a free-living wild animal that has been confirmed by emm gene sequencing and the first report of the emm 28 strain in a nonhuman species.

Prior to the availability of molecular techniques in the 1970s, identification of $S$. pyogenes was performed through the assessment of biochemical characteristics combined with Lancefield grouping. This is considered an inaccurate sole means of identification and is now conducted in conjunction with gene sequencing of the M-protein antigen, known as emm typing (Facklam, 2002). Infection with S. pyogenes has been reported in wild rodents in the USA (Bell et al., 1958) in which it was associated with disseminated abscessation but as this study pre-dated moleculartyping, these results are unconfirmed. Streptococcus pyogenes infection has previously been reported in domestic animals including dogs and cats in Europe and the USA (Greene, 2011; Weese and Fulford, 2011) and in cattle in 
Denmark (Henningsen and Ernst, 1937) and Canada ( Pullin, 1947). Clinical disease in dogs and cats has not been reported except for one equivocal case of canine conjunctivitis (Sprot et al., 2012). Reports of infection in farmed cattle have been associated with mastitis (Pullin, 1947; Henningsen and Ernst, 1937). Disease associated with the bacterium has also been recorded in captive wild animals that exist in close contact with humans, including a laboratory rhesus monkey (Macaca mulatta) with streptococcal toxic shock syndrome (García et al., 2006) and farmed white-tailed deer (Odocoileus virginianus) with pneumonia (Whitlock, 1939), both reported in the USA.

Until the current study, $S$. pyogenes emm 28 had not been reported from any non-human species. In humans, emm 28 strain can be associated with both superficial and invasive disease and accounts for a third of the cases of puerperal sepsis in Europe (Luca-Harari et al., 2009). In the UK, reports of human cases are sporadic with no reported seasonal or spatial pattern of infection (Public Health England, unpublished data).

In humans, $S$. pyogenes is transmitted via aerosol-inhalation, skin and mucous membrane exposure to nasal, pharyngeal or wound secretions and, rarely, via contaminated food sources ( Boston University Research Compliance, 2012). Whilst we cannot exclude that the hedgehog contracted $S$. pyogenes infection from another (non-human) animal, such as a domestic pet or a mastitic cow, the rarity of described non-human infections indicates that this is unlikely. We postulate that infection in this case occurred due to anthroponotic transmission.

Direct human-hedgehog contact frequently occurs in wildlife rehabilitation centers and is a known route of exposure for people to zoonotic diseases (Riley and Chomel, 2005). Indirect contact with hedgehogs may occur within gardens and although fomite transmission is not thought to be a common source of $S$. pyogenes infection in humans, transmission via this route remains a possibility.

The frequency of interactions between humans, domestic animals and wildlife is increased by the anthropogenic loss of wildlife habitat which augments the risk of these groups being exposed to novel pathogens (Daszak et al., 2000; Epstein and Price, 2009). Many studies have focused on zoonotic diseases originating from wildlife but relatively few have concerned anthroponoses (otherwise known as reverse zoonoses), where human pathogens adversely affect captive or wild animals (Messenger et al., 2014). In addition to being a threat to wildlife health, there is the possibility that anthroponoses may spillback to humans (Henningsen and Ernst, 1937). This report of GAS infection in a hedgehog may be an isolated case, but further research on the pathogens affecting wildlife is required to better understand any disease threats to conservation or public health.

\section{References}

Boston University Research Compliance (2012) Boston: Research Occupational Health Program Agent Information Sheet- Streptococcus pyogenes; 2012. Available: http://www.bu.edu/rohp/files/2012/08/Streptococcus-pyogenes.pdf [accessed January 9, 2015]

Bell JF, Owen CR, Jellison WL (1958) Group A streptococcus infections in wild rodents. Journal of Infectious Disease 103: 196-203

Cunningham MW (2000) Pathogenesis of group A streptococcal infections. Clinical Microbiology Reviews 13: 470-511

Daszak P, Cunningham AA, Hyatt AD (2000) Emerging infectious diseases of wildlife threats to biodiversity and human health. Science287: 443-9. 
Epstein JH, Price JT (2009) The significant but understudied impact of pathogen transmission from humans to animals. Mount Sinai Journal Of Medicine 76: 448- 55; DOI:10.1002/MSJ [Online March 20, 2015]

Facklam, R (2002) What happened to the streptococci: overview of taxonomic and nomenclature changes. Clinical Microbiology Reviews 15: 613-30

García A, Paul K, Beall B, Mcclure H (2006) Toxic shock due to Streptococcus pyogenes in a rhesus monkey (Macaca mulatta). Journal of the American Association for Laboratory Animal Science 45: 79-82

Greene CE (2011) Gram-positive bacterial infections. In: Infectious Disease of the Dog and Cat (4th ed), CE Greene (editor), St. Louis: Elsevier, pp 325-328

Harris S, Morris P, Wray S, Yalden D (1995) A review of British mammals $\square$ : population estimates and conservation status of British mammals other than cetaceans, Peterborough: JNCC, p 216

Henningsen EJ, Ernst $J$ (1937) Milk epidemic of angina, originating from a cow with mastitis and due to Streptococcus pyogenes (Lancefield group A). The Journal of Hygiene 38: 38491

Lamagni TL, Neal S, Keshishian C, Alhaddad N (2008) Severe Streptococcus pyogenes infections, United Kingdom. Emerging Infectious Diseases 14: 201-209

Luca-Harari B, Darenberg J, Neal S, Siljander T, Strakova L, Tanna A, et al. (2009) Clinical and microbiological characteristics of severe Streptococcus pyogenes disease in Europe. Journal of Clinical Microbiology 474: 1155-65; DOI:10.1128/JCM.02155-08 [Online March $20,2015]$

Meredith AL, Milne EM (2009) Cryptosporidial Infection in a Captive European Hedgehog ( Erinaceus europaeus ). Journal of Zoo and Wildlife Medicine 40: 809-811

Messenger AM, Barnes AN, Gray GC (2014) Reverse zoonotic disease transmission (zooanthroponosis): a systematic review of seldom-documented human biological threats to animals. PloS One9: e89055; DOI:10.1371/journal.pone.0089055 [Online March 20, 2015]

Pullin JW (1947) An outbreak of mastitis caused by Streptococcus pyogenes and the effect of treatment with penicillin. Canadian Journal of Comparative Medicine 11: 45-46

Riley PY, Chomel BB (2005) Hedgehog zoonoses. Emerging Infectious Diseases 11: 1-5

Robinson IAN, Routh A (1999) Veterinary care of the hedgehog. In Practice 21: 128-137

Skuballa J, Oehne R, Hartelt K, Petney T, Bücher T, Kimmig P, Taraschewski H (2007) European hedgehogs as hosts for Borrelia spp. Emereging Infectious Diseases 13: 952-953

Sprot H, Efstratiou A, Hubble M, Morgan M (2012) Man's best friend?--first report of prosthetic joint infection with Streptococcus pyogenes from a canine source. Journal of Infection 64: 625-7

Wembridge D (2011) The state of Britain's hedgehogs. Available: http://www.britishhedgehogs.org.uk/leaflets/sobh.pdf [accessed October 14, 2014] 
Whitlock SC (1939) The prevalence of disease and parasites in whitetail deer. In: Transactions, North American Wildlife Conference, p. 244-249

Widen F, Gavier-Widen D, Nikiila T, Morner T (1996) Fatal herpesvirus infection in a hedgehog (Erinaceus europaeus). The Veterinary Record 139: 237-238 